\title{
Zur Rolle von Bibliotheken in digitalen Forschungsinfrastrukturen
}

\section{Wissenschaftspolitisches Umfeld}

Der Begriff „Digitalisierung“ ist seit einigen Jahren in aller Munde - in Deutschland hat die Bundesregierung die „Digitale Agenda 2014-2017“ ausgerufen. ${ }^{1}$ Die Handlungsfelder reichen in alle Lebensbereiche unserer Gesellschaft: Infrastrukturen, Wirtschaft und Arbeiten, innovativer Staat, Lebenswelten, Bildung, Forschung, Wissenschaft, Kultur und Medien, Sicherheit, europäische und internationale Dimensionen. Betrachtet man das Handlungsfeld der Wissenschaft, so nimmt sich das Bundesministerium für Bildung und Forschung (BMBF) einer federführenden Rolle an, indem weitere Ziele definiert werden: den digitalen Wandel in der Wissenschaft forcieren, Zugang $\mathrm{zu}$ Wissen als Grundlage für Innovation sichern, Bildungsoffensive für die digitale Wissensgesellschaft, Innovationspotenziale der Digitalisierung nutzen, durch Forschung den digitalen Wandel verstehen sowie Kultur und Medien als Teile des Handlungsfeldes begreifen. ${ }^{2}$

Wenngleich sich die Geschichte der Informationsinfrastrukturen bis in die 1960er Jahre zurückverfolgen lässt, ${ }^{3}$ gab es wesentliche Entwicklungen vornehmlich in den letzten Jahren. Der Wissenschaftsrat veröffentlichte bereits 2012 Empfehlungen für die Entwicklungen der wissenschaftlichen Informationsinfrastrukturen in Deutschland, ${ }^{4}$ die starke digitale Komponenten beinhalten. Die aufgrund der Länderverantwortungen für Wissenschaft bedeutende Gemeinsame Wissenschaftskonferenz des Bundes und der Länder (GWK) fasste im Jahr 2013 einen Beschluss zum Aufbau eines Rates für Informationsinfrastrukturen (RfII), der sich dann im Herbst 2014 konstituierte. $^{5}$ In seinem umfassende-

1 Presse- und Informationsamt der Bundesregierung: Digitale Agenda 2014-2017. https:// www.digitale-agenda.de/Webs/DA/DE/Home/home_node.html (23.1.2018).

2 Bundesministerium für Bildung und Forschung: Die Digitale Agenda - relevant auch für Bildung, Wissenschaft und Forschung, 2014. https://www.bmbf.de/de/die-digitale-agenda-relevant-auch-fuer-bildung-wissenschaft-und-forschung-206.html (23.1.2018).

3 Rat für Informationsinfrastrukturen: Die Entwicklung von Konzepten für Informationsinfrastrukturen in der Bundesrepublik Deutschland seit den 1960er Jahren. Göttingen, Juni 2016. http://www.rfii.de/?wpdmdl=2041 (24.1.2018).

4 Wissenschaftsrat: Empfehlungen zur Weiterentwicklung der wissenschaftlichen Informationsinfrastrukturen in Deutschland bis 2020. Berlin, 13. Juli 2012. http://www.wissenschaftsrat.de/download/archiv/2359-12.pdf (23.1.2018).

Ә Open Access. () 2018 Wolfram Horstmann, publiziert von De Gruyter. (G) BY-NC-ND Dieses Werk ist lizenziert unter der Creative Commons Attribution-NonCommercial-NoDerivatives 4.0 Lizenz. 
ren Auftrag widmet sich der RfII mit hoher Priorität dem Thema Forschungsdaten, das er als große Herausforderung der Wissenschaft hervorhebt. ${ }^{6}$ Die Allianz der Wissenschaftsorganisationen zeichnet in ihrer Schwerpunktinitiative „Digitale Information“ für die Jahre 2018-2022 ein weiter aufgefächertes Bild, das acht Themenfelder in den Blick nimmt: Publikationen, Werkzeuge, IT, Datensammlungen, Personal, Lehre, Recht und allgemeine Wissenschaftspraxis. ${ }^{7}$

Auf europäischer Ebene wurde das Thema digitaler Forschungsinfrastrukturen politisch in inhaltlich und zeitlich ähnlicher Weise wie in Deutschland entwickelt. Ein maßgeblicher, allgemeiner Impuls ging 2015 von der Initiative für einen digitalen Binnenmarkt („Digital Single Market“) aus, der gesellschaftsübergreifend ausgerichtet ist. ${ }^{8}$ Innerhalb der Handlungslinien für die Digitalisierung der Wirtschaft („Digitising European Industry“) wird unter anderem die „European Cloud Initiative“ verfolgt, die zum Ziel hat, die Wettbewerbsfähigkeit datengetriebener Aktivitäten in Europa zu erhöhen. Neben dem Hochleistungsrechnen (High Performance Computing) und Investitionen in eine Dateninfrastruktur (European Data Infrastructure) wird die European Open Science Cloud (EOSC) ausgerufen, die speziell wissenschaftliche Belange anspricht.

Auch global spielen diese Themen zunehmend eine wichtigere Rolle. Die UN adressiert sie in den „Sustainable Development Goals“: „The spread of information and communications technology and global interconnectedness has great potential to accelerate human progress, to bridge the digital divide and to develop knowledge societies, as does scientific and technological innovation across areas". ${ }^{9} \mathrm{Zu}$ den bestehenden weltweiten Initiativen wie CoData ${ }^{10}$ gesellen sich neue weltweit vernetzte Foren wie die Research Data Alliance, ${ }^{11}$ die spezifische wissenschaftliche Belange behandeln.

5 Gemeinsame Wissenschaftskonferenz: Rat für Informationsinfrastrukturen, 2014. http:// www.gwk-bonn.de/themen/uebergreifende-wissenschafts-und-forschungspolitische-themen/ informationsinfrastruktur/ (23.1.2018).

6 RfII, Entwicklung (wie Anm. 3).

7 Steuerungsgremium der Schwerpunktinitiative „Digitale Information“ der Allianz der Wissenschaftsorganisationen: Den digitalen Wandel in der Wissenschaft gestalten. Die Schwerpunktinitiative „Digitale Information“ der Allianz der deutschen Wissenschaftsorganisationen. Leitbild 2018-2022, Dezember 2017. http://doi.org/10.2312/allianzoa.015.

8 European Commission: Mid-Term Review on the implementation of the Digital Single Market Strategy. A Connected Digital Single Market for All. Brüssel, 10. Mai 2017. http://eur-lex. europa.eu/legal-content/EN/TXT/?uri=CELEX:52017DC0228 (8.2.2018).

9 United Nations: Sustainable Development Goals. https://sustainabledevelopment.un.org/ post2015/transformingourworld (8.2.2018).

10 Committee on Data of the International Council for Science: CODATA's Mission. http:// www.codata.org/about-codata/our-mission (8.2.2018).

11 Research Data Alliance: About RDA. https://www.rd-alliance.org/about-rda (8.2.2018). 


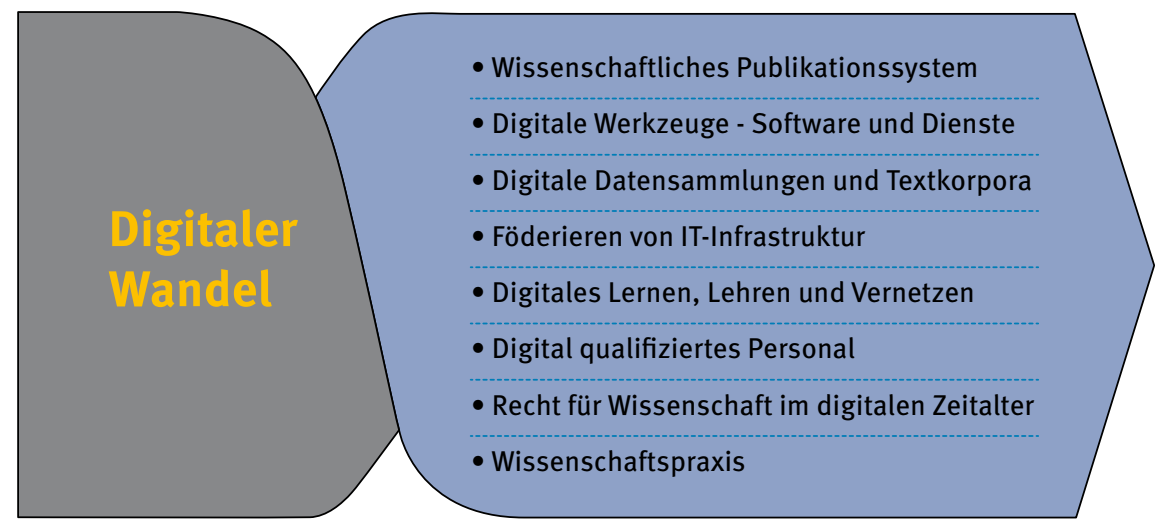

Abb. 1: Handlungsfelder nach dem Leitbild 2018-2022 der Schwerpunktinitiative „Digitale Information“ der Allianz der deutschen Wissenschaftsorganisationen.

\section{Das Thema Digitalisierung in wissenschaftlichen Bibliotheken}

Der Begriff Digitalisierung ist in wissenschaftlichen Bibliotheken bereits seit Mitte der 1990er Jahre etabliert. ${ }^{12}$ Der Bedarf digitaler Informationsinfrastrukturen wurde schon 1995 vom Bibliotheksausschuss und der Kommission für Rechenanlagen der Deutschen Forschungsgemeinschaft (DFG) als Desiderat benannt. Meist wurde Digitalisierung jedoch mit Retrodigitalisierung gleichgesetzt - also der Überführung analoger, gedruckter Materialien in ein digitales Format mittels Bildgebung, Texterkennung oder Transkription und Veröffentlichung im Internet. Digitalisierung als gesamtgesellschaftliches Phänomen zu betrachten, kann daher durchaus als überraschend empfunden worden sein. In der Tat hat sich aber der Aufgabenzuschnitt der Bibliotheken in den letzten 20 Jahren drastisch verändert. So formuliert der europäische Verband für wissenschaftliche Bibliotheken LIBER in seiner Strategie für die Jahre 2018-2022 ${ }^{13}$ die zentralen Ziele, (1) innovative Wissenschaftskommunikation voranzutreiben, (2)

12 Bund-Länder-Kommission für Bildungsplanung und Forschungsförderung: Digitalisierung von wissenschaftlichen Bibliotheken. Bonn 2000. http://www.blk-bonn.de/papers/heft84.pdf (8.2.2018).

13 LIBER Europe: Research Libraries Powering Sustainable Knowledge in the Digital Age. Den Haag, 16. November 2017. http://libereurope.eu/wp-content/uploads/2017/11/LIBER-Strategy2018-2022.pdf (5.2.2018). 
als Knoten für digitale Fertigkeiten in der Wissenschaft zu fungieren und (3) gemeinschaftlich mit der Wissenschaft digitale Forschungsinfrastruktur auszubauen. Diese Ziele stehen ebenfalls im Zentrum der wissenschaftlichen Aspekte der Digitalen Agenden von Bundesregierung und Europäischer Union sowie der neuen Strategie der Allianz der Wissenschaftsorganisationen (s. o.). Selbst im Weltverband der öffentlichen Bibliotheken IFLA wird die Bedeutung dieser Themen hervorgehoben: Bereits zwei der vierteljährlich erscheinenden Ausgaben der Verbandszeitschrift IFLA Journal widmeten sich den Forschungsdaten. ${ }^{14}$ Diese zusammengenommen sehr prominente Rolle der Bibliotheken in der Digitalisierung würdigte auch die Bundesministerin für Bildung und Forschung, die Bibliotheken vor allem mit aufklärerischen Aufgaben im digitalen Zeitalter in Verbindung bringt. ${ }^{15}$

\section{Der Gegenstandsbereich „Digitale Forschungs- infrastrukturen“}

Wie bereits deutlich wurde, ist die Begriffsvielfalt und die dahinterstehende konzeptionelle Komplexität im Gegenstandsbereich digitaler Forschungsinfrastrukturen beträchtlich. Hinzu kommt, dass viele englische Begriffe verwendet werden. Um eine Abgrenzung vorzunehmen, seien einige Begriffe hier eingeführt. Diese Betrachtung ist keinesfalls vollständig und kann nur eine Annäherung darstellen.

Digitale Forschungsinfrastrukturen sind eine Grundlage für die Analyse und Interpretation natürlicher und kultureller Phänomene, in der Forschende digitale Methoden und Wissensrepräsentationen anwenden. Sie sind also ein Bestandteil digitaler Forschung (Digital Research). Dies kann eine Klimaforscherin sein, die digitale Daten über Wetteraufzeichnungen verarbeitet und Modellsimulationen erstellt, oder ein Historiker, der Digitalisate von Dokumenten aus dem 18. Jahrhundert in einer digitalen Edition annotiert. Zur Verdeutlichung kann man Digital Science (Digitale Forschung in den Naturwissenschaften) und Digital Humanities (Digitale Forschung in den Geisteswissenschaften) gegenüberstellen. Dabei ist auch zu beobachten, dass - obwohl Science im englischen Sprachgebrauch eine Konnotation der Naturwissenschaften trägt - die

14 Witt, Michael u. Wolfram Horstmann: International approaches to research data services in libraries. In: IFLA Journal 42 (2016) H. 4. S. 251-252.

15 Vgl. Wanka, Johanna: Bibliotheken sind zentrale Akteure der Digitalisierung. In: BuB Forum Bibliothek und Information 69 (2017) H. 7. S. 397-399. 
gesamte digitale Forschung unter Digital Science subsumiert wird. Eine Definition der Europäischen Kommission beispielsweise macht keine Unterscheidung zwischen Sciences und Humanities, schließt aber wohl die Geisteswissenschaften ein: „Digital science means a radical transformation of the nature of science and innovation due to the integration of ICT in the research process and the internet culture of openness and sharing."16

Die Übergänge zwischen digitaler und analoger Forschung sind fließend, sodass die oben erwähnte Klimaforscherin oder der Historiker sich im Regelfall nicht als Digital Researcher oder Analog Researcher bezeichnen würden. Dies wird auch durch die Begriffe „eResearch“ und „eScience“ deutlich, bei denen das „e“ nicht unbedingt für electronic (wie in eLearning oder eCommerce), sondern für enhanced stehen kann, um die Erweiterung der Wissenschaft durch jegliche Art neuer Methoden und Wissensrepräsentationen sowie den fließenden Übergang digitaler und analoger Aspekte in den Vordergrund zu stellen.

Die Gegenüberstellung der Geisteswissenschaften mit den Bereichen Naturwissenschaft, Technik und Medizin (STEM: Science, Technology, Engineering, Medicine) ist hilfreich, um eine Abgrenzung vorzunehmen, jedoch sind die Übergänge ebenfalls fließend. Es gibt ebenso gut Forschende in den Geisteswissenschaften, die mit digitalen Methoden naturwissenschaftliche Phänomene untersuchen (etwa in der Wissenschaftsgeschichte), wie Forschende in den Naturwissenschaften digitale Methoden und Wissensrepräsentationen der Geisteswissenschaften analysieren (etwa historische Karten zu Vulkanen oder Wetteraufzeichnungen). Besonders das emporkommende Feld der Datenwissenschaften (Data Science) kann eine agnostische Position gegenüber den traditionellen disziplinären Grenzen einnehmen, wenn der Fokus in der Tat auf Daten jeglicher Herkunft gelegt wird. Wenn anstatt auf Algorithmen strikt auf Daten aller Art geblickt wird, stärkt dies auch die Abgrenzung gegenüber der Informatik, die Algorithmen bereits in ihrem Forschungsprofil integriert hat. Derzeit allerdings ist in der Datenwissenschaft wohl eher ein Überhang zu den STEMDisziplinen und eine starke Fokussierung auf die Informatik zu beobachten.

Schließlich finden auch die Sozialwissenschaften in dieser vereinfachten Gegenüberstellung keine Berücksichtigung. Digitale Methoden und Wissensrepräsentationen sind jedoch hier ebenso von besonderer Bedeutung. Bilden doch statistische Werkzeuge in der quantitativen Sozialforschung und den Wirtschaftswissenschaften, Videoanalyse in der qualitativen Sozialforschung und

16 European Commission: Digital science in Horizon 2020, März 2013. http://ec.europa.eu/ newsroom/dae/document.cfm?doc_id=2124 (5.2.2018). 
auch seit Dekaden fest etablierte Datenzentren für Längsschnittstudien und Bevölkerungsumfragen feste Bestandteile der sozialwissenschaftlichen Forschungstätigkeiten.

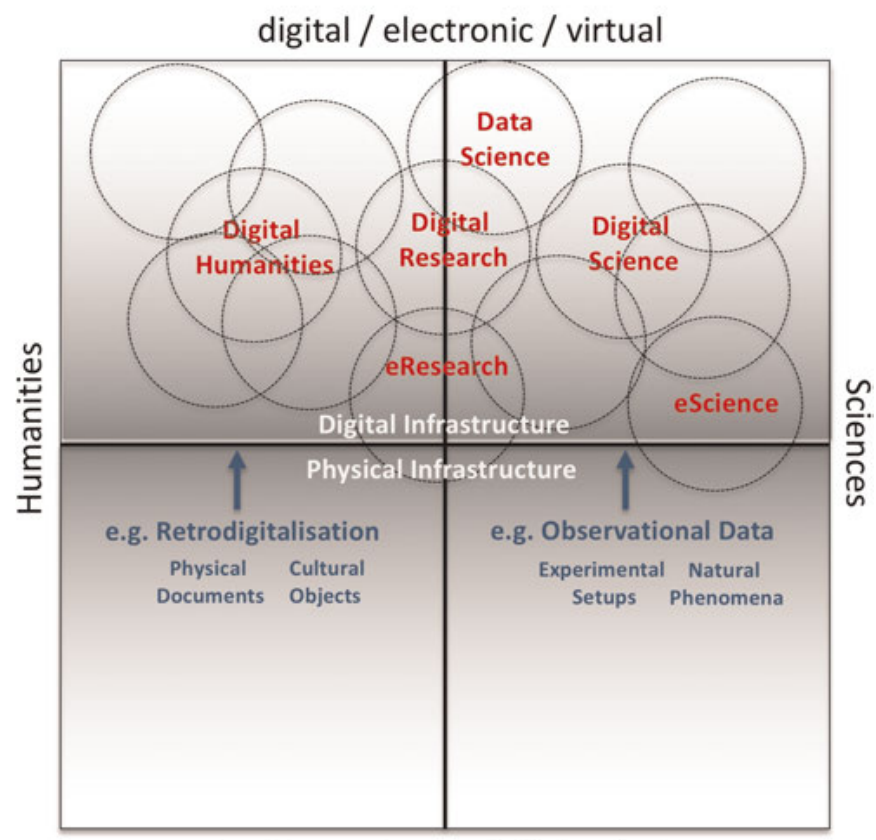

analog / non-electronic / physical

Abb. 2: Vereinfachte Gegenüberstellung von Begriffen zur digitalen Infrastruktur im Kontext der Geistes- und Naturwissenschaften sowie der analogen und digitalen Welt; eine erklärende und kritische Betrachtung erfolgt im Text.

\section{Digital Humanities und Digital Science}

Trotz der fließenden Übergänge zwischen digitaler und analoger Forschung sowie zwischen den Geistes- und Naturwissenschaften haben sich Stereotypen ausgebildet, die sich strukturell im Wissenschaftssystem niederschlagen. Professuren für Digital Humanities (s. u.) und Institute und Studiengänge für Data Science sind beispielsweise in den letzten Jahren an Universitäten verstärkt eingerichtet worden. 
Die Digital Humanities sind ein junges, aufstrebendes Feld, das bereits über umfassende Bibliografien $\mathrm{zu}$ beschreiben ist ${ }^{17}$ und zunehmend Professuren erhält. ${ }^{18}$ Es hebt sich durch seine starke (auch quantitative) Methodenorientierung von traditionellen Geisteswissenschaften ab. Die genaue Definition wird intensiv diskutiert, ${ }^{19}$ ebenso wie Grundsatzfragen in der Beziehung zu traditionellen Geisteswissenschaften: Sind digitale Methoden eine eigene Forschungsfrage oder lediglich ein Hilfsmittel? Ist das Digitale ein in seiner Gesamtheit eigener Kulturgegenstand? Sollten Digital Humanities die Naturwissenschaft kontextualisieren, die ihrerseits immer mehr auf Daten basiert und Gefahr läuft, verständliche Narrative dafür zu verlieren, was diese für den „Geist“ und die Menschheit bedeuten? Durch die massive Verwendung von Dokumenten und Objekten (Wissensrepräsentationen) aus dem Kulturgut ist eine enge Verbindung der Digital Humanities mit den Bibliotheken, Archiven und Museen und der Retrodigitalisierung offensichtlich. Generell kann man formulieren: Die Digitalisierung von Bibliotheken, Archiven und Museen (im allgemeinen Sinne) liefert die digitale Infrastruktur für die Geisteswissenschaften (s. u.).

In den STEM-Fächern stehen anstelle des Kulturguts vor allem Beobachtungsdaten zu Naturphänomenen und Experimente im Zentrum der Betrachtung digitaler Forschungsinfrastruktur. Für die ausgeprägte Bindung zu Experimentalgeräten und Experimentalsoftware sowie Datenrepositorien existieren viele Beispiele, etwa Maschinen zur Gensequenzierung in der Molekularbiologie, Teleskope in der Astronomie, Sensorennetzwerke in der Klimaforschung, Forschungsschiffe in der Meeresforschung, Teilchenbeschleuniger in der Hochenergiephysik, die eine hohe Datenproduktion sowie eine ausgeprägte Nutzung und Produktion von Software und die Erstellung von Computermodellen und deren Simulation mit sich bringen. Strukturell zeigt sich dies im Wissenschaftssystem in zahlreichen „Bindestrich-Informatikern“ (Bio-Informatik, Geo-Informatik etc.) und internationalen Forschungsinfrastrukturen wie dem CERN oder genuin digital angelegten Zentren wie dem Deutschen Klimarechenzentrum (DKRZ) oder dem European Bioinformatics Institute (EBI). Man könnte der digitalen Infrastruktur in den STEM-Fächern eine größere Bindung zu den über Rechenzentren bereitgestellten Speicher- und Rechenkapazitäten zuschreiben, ebenso wie den Geisteswissenschaften eine Bindung zu den Bibliotheken, Ar-

17 DARIAH Deutschland: Doing Digital Humanities, 2018. https://de.dariah.eu/bibliographie (5.2.2018).

18 Sahle, Patrick: Zur Professoralisierung der Digital Humanities, 23. März 2016. http://dhdblog.org/?p=6174 (5.2.2018).

19 Heppler, Jason: What is Digital Humanities? 2015. https://whatisdigitalhumanities.com (5.2.2018). 
chiven und Museen. Dieses Stereotyp wird am Ende dieses Textes differenzierter betrachtet.

Digitale Infrastruktur kann je nach Fach sehr unterschiedliche Ausprägungen aufweisen. Während das CERN als Quelle einzigartiger Daten ein weltweites Netzwerk sternförmig vom Zentrum in die Peripherie anlegen kann, ${ }^{20}$ muss ein Klimarechenzentrum zunächst weltweit Daten aus der Welt integrieren. ${ }^{21}$ In den Geisteswissenschaften hingegen erkennt man etwa bei DARIAH eine Netzwerkstruktur zwischen einer großen Anzahl lokal verorteter Einzelforscher oder Arbeitsgruppen. ${ }^{22}$

Die betriebliche Verortung digitaler Infrastruktur in der Wissenschaft ist über alle Fächer hinweg uneinheitlich. Zum Teil spielen Bibliotheken und Rechenzentren in Universitäten eine maßgebliche Rolle, zum Teil werden diese in außeruniversitären Forschungseinrichtungen bereitgestellt, in Deutschland beispielsweise in der Helmholtz-Gemeinschaft, zum Teil sind es einzelne Lehrstühle, Labore oder Arbeitsgruppen, die direkt auf kommerzielle Dienste für Speicher- und Rechenkapazität und eine große Vielfalt digitaler Werkzeuge zurückgreifen. ${ }^{23}$

\section{„Openness“ - das Thema Offenheit}

Eine weitere Dimension digitaler Infrastrukturen ist das Thema Offenheit in akademischen Kulturen. Wie das oben genannte Beispiel der Definition von Digital Science der Europäischen Kommission zeigt, wird es im Kontext der Digitalisierung häufig mit Fragen der Wissenschaft und der Infrastruktur (,internet culture of openness and sharing“) verwoben. Grundsätzlich geht es um die entgeltfreie und für alle Interessenten zugängliche Nutzung von Objekten in der Wissenschaft und Aktivitäten, die auf eine offene Nutzung ausgerichtet sind

20 I. Ueda and the ATLAS collaboration: ATLAS Operations: Experience and Evolution in the Data Taking Era. In: Journal of Physics: Conference Series 331 072034, 2011.

21 Bernholdt, D. u. a.: The Earth System Grid: Supporting the Next Generation of Climate Modeling Research. In: Proceedings of the IEEE 93 (2005) H. 3. S. 485-495.

22 Blümm, Mirjam u. a.: Vom Projekt zum Betrieb: Die Organisation einer nachhaltigen Infrastruktur für die Geisteswissenschaften DARIAH-DE. In: ABI Technik 36 (2016) H. 1. S. 10-23. http://www.degruyter.com/view/j/abitech.2016.36.issue-1/abitech-2016-0011/abitech-20160011.xml (24.4.2016).

23 Kramer, Bianca u. Jeroen Bosman: Innovations in Scholarly Communication, 2016. https:// 101innovations.wordpress.com (5.2.2018); Wolski, Malcolm, Louise Howard u. Joanna Richardson: The importance of tools in the data lifecycle. In: Digital Library Perspectives 33 (2017) H. 3. S. $235-252$. 
(vgl. Abb. 3). Es würde den Rahmen dieser Darstellung sprengen, jeden einzelnen Aspekt der Offenheit zu diskutieren. Daher seien zunächst nur die Oberbegriffe eingeführt. Open Access bezieht sich vor allem auf wissenschaftliche Literatur, während Open Data für das Forschungsdatenmanagement von Bedeutung ist. Es gibt deutliche Unterschiede in der Reichweite der Forderungen nach Offenheit, da Open Access zum Ziel hat, die komplette wissenschaftliche Literatur freizustellen. Hingegen werden im Forschungsdatenmanagement Grenzen gezogen, darunter fallen der Schutz der Privatsphäre bei Nutzerdaten, Sicherheit (etwa bei Waffensystemen), wirtschaftliche Interessen oder das Interesse der einzelnen Forschenden, Daten zunächst selbst zu verstehen, auszuwerten und darüber zu publizieren, bevor sie geteilt werden. Zudem ist Open Access bereits seit 20 Jahren in der Diskussion und zeigt wissenschaftlich und wirtschaftlich Strukturbildung, etwa in institutionalisierten Dokumentenservern und Services oder Open-Access-Zeitschriften, ${ }^{24}$ während der Gegenstand Forschungsdaten aktuell sehr intensiv behandelt wird. ${ }^{25}$ Noch weiter zurück reicht das Thema der Open-Source-Software, ${ }^{26}$ das wiederum im Zusammenhang mit Forschungsdatenmanagement an Bedeutung gewinnt (s. o.). Für die Aktivitäten, die zur Offenheit beitragen, kann eine grundsätzliche Unterscheidung zwischen Forschung (Open Science) und Lehre (Open Education) Anwendung finden. Wenn wirtschaftliche Interessen betroffen sind, wird häufiger der Begriff Open Innovation verwendet, da der Produktentwicklungszyklus durch Teilung von Investitionen beschleunigt werden kann. Ein Beispiel ist das Structural Genomics Consortium, ${ }^{27}$ das Forschung für die Pharmaentwicklung durch gemeinsame Investitionen der Firmen bündelt, die somit redundante Aktivitäten in ihren eigenen Forschungsabteilungen verhindern und die Offenheit in der Patententwicklung erhöhen.

24 Horstmann, Wolfram: From collecting to connecting - the role of libraries in Open Access. In: Praxishandbuch Open Access. Hrsg. von Konstanze Söllner u. Bernhard Mittermaier. Berlin, Boston: de Gruyter 2017. S. 62-74. http://www.degruyter.com/view/books/9783110494068/ 9783110494068-008/9783110494068-008.xml (5.2.2018).

25 Rat für Informationsinfrastrukturen: Leistung aus Vielfalt. Empfehlungen zu Strukturen, Prozessen und Finanzierung des Forschungsdatenmanagements in Deutschland. Göttingen 2016. http://www.rfii.de/?wpdmdl=1998 (21.1.2018).

26 Bretthauer, David: Open Source Software: A History, 2001 (Published Works). http://digitalcommons.uconn.edu/libr_pubs/7 (5.2.2018).

27 Alexy, Oliver, Paola Criscuolo u. Ammon Salter: Does IP Strategy Have to Cripple Open Innovation? In: MIT Sloan Management Review 51 (2009) H. 1. S. 71-77. 


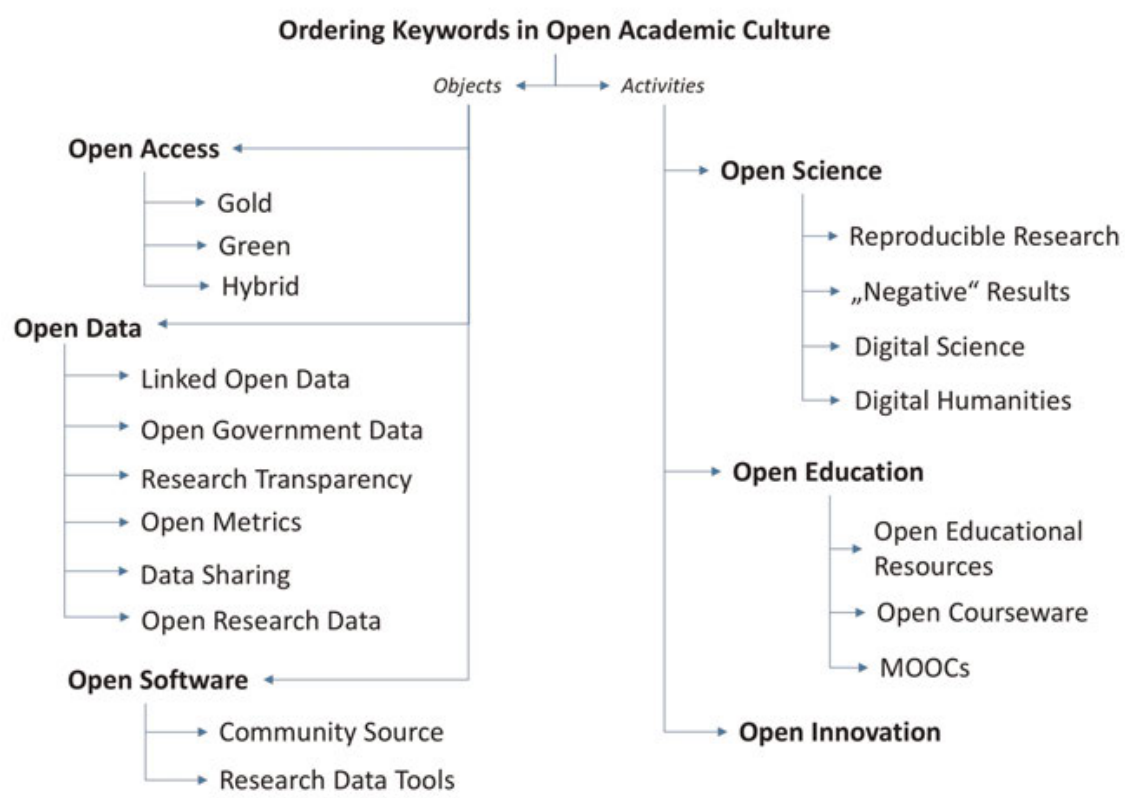

Abb. 3: Vereinfachte Gegenüberstellung von Begriffen zur Offenheit in der Wissenschaft.

\section{Kooperativität in verteilten Infrastrukturen}

Forschung behandelt prinzipiell jeden einzelnen Aspekt von Natur, Gesellschaft und Menschheit. Allein aufgrund der Masse an verfügbaren Informationen muss das Vermitteln und Erzeugen von Wissen über viele Experten und Institutionen verteilt werden. Um diese verteilte Spezialisierung zusammenzufügen, kooperieren Experten und Institutionen intensiv. Nicht nur die Forschung selbst, sondern auch deren Infrastruktur ist daher verteilt und kooperativ angelegt. Infrastruktur ist jedoch zugleich ein Konzentrat der Gemeinsamkeiten von Forschung: Forschende greifen z. B. gemeinsam auf Wissensbestände wie Bücher und Zeitschriften zu, die von Bibliotheken bereitgestellt werden, oder nutzen Netzwerke, Speicher- und Rechenkapazität, die von Rechenzentren bereitgestellt werden. Betrachtet man vereinfachend nur digitale Wissensbestände in Bibliotheken und Dienste in Rechenzentren, stellen Bibliotheken und Rechenzentren kooperativ digitale Forschungsinfrastruktur für viele fachlich ausdifferenzierte Spezialgebiete der Forschung zur Verfügung. 
Die konkreten Ausprägungen der digitalen Forschungsinfrastruktur sind aber weitaus komplexer. Zunächst ist das Verhältnis zwischen Bibliotheken und Rechenzentren nicht einheitlich und es gibt außer diesen beiden noch viele weitere Akteure für fachübergreifende digitale Infrastruktur: neben Verwaltungen, Archiven und Museen im öffentlichen System auch privatwirtschaftliche Verlage und IT-Dienstleister (s. Abschnitt „Chancen und Risiken für wissenschaftliche Bibliotheken in digitalen Forschungsinfrastrukturen“). Zum anderen ist das Verhältnis zwischen fachübergreifenden und fachspezifischen digitalen Infrastrukturen vielfältig. Die Anforderungen der einzelnen Fächer an digitale Infrastruktur leiten sich aus den jeweiligen Forschungsfragen und Forschungsmethoden ab und weisen deutliche Unterschiede auf. Daher sind den Gemeinsamkeiten, die Bibliotheken und Rechenzentren abbilden können, Grenzen gesetzt. Die Anforderungen beziehen sich auch nicht notwendigerweise auf das Digitale (Virtuelle), sondern auf die physikalischen Bedingungen der Forschung, also Büros, Labore oder Großgeräte. Die Beschaffenheit der physikalischen Forschungsinfrastruktur hat maßgeblichen Einfluss auf die Anforderungen an die digitale Forschungsinfrastruktur und bedingt, dass sich sehr unterschiedliche Muster in ihrer Gestaltung ausprägen. Wie zuvor erwähnt, werden die Hochenergiephysik oder die Klimaforschung auf Speichenmodelle um zentrale Knoten (im CERN und verbundenen Zentren) setzen, während in den Geisteswissenschaften auf Verbindungen zwischen Endknoten (also einzelne Forschende und Projekte) abgezielt wird.

\section{Digitale Forschungsinfrastrukturen in Deutschland und Europa}

Die Digitalisierung der Forschungsinfrastrukturen befindet sich in der Umsetzung. Den diversen Einflüssen aus Forschung und Infrastrukturen entsprechend, bilden sich vielfältige Initiativen und Organisationsmodelle heraus. Eine Gegenüberstellung von Beispielen aus Deutschland und Europa gewährt einen Einblick in diese Vielfalt (vgl. Tab. 1, die auch alle Referenzen auf weitere Informationen für diesen Abschnitt enthält). Aus Sicht der (nicht genuin digitalen) Forschungsinfrastrukturen ist in Deutschland beispielweise die Roadmap für Forschungsinfrastrukturen des BMBF zu nennen, die eine europäische Entsprechung in der Roadmap des European Strategy Forum on Research Infrastructures (ESFRI) hat. Für digital orientierte Rahmenkonzepte befindet sich in 
Deutschland die Nationale Forschungsdateninfrastruktur (NFDI) in der Entwicklung, während es in Europa die EOSC ist.

Einige Unterschiede zwischen NFDI und EOSC sind hervorzuheben. Die NFDI ist zunächst ein Konzept, das den wissenschaftspolitischen Prozess der Umsetzung begleitet. Das NFDI-Konzept schlägt vor, digitale Infrastruktur über die Grenzen von Bund und Ländern, zwischen Universitäten und außeruniversitären Forschungseinrichtungen sowie zwischen den verschiedenen Wissenschaftsorganisationen kooperativ und verteilt in Konsortien aufzustellen und stellt das Primat der wissenschaftlichen Leitung heraus. Dem folgend wird auch keine Umsetzung als einzelne Infrastruktur, sondern als mehrere fachspezifische Infrastrukturen vorgeschlagen. Es ist damit zu rechnen, dass der GWK ein Konzept zur Ausgestaltung und Finanzierung vorgelegt wird.

Während also in Deutschland Steuerung und Finanzierung zuerst beschlossen werden, wird die EOSC bereits im Zuge des laufenden Forschungsrahmenprogrammes Horizon 2020 in einigen Pilotprojekten entwickelt und parallel eine langfristige Förderung, Steuerung und infrastrukturelle Verankerung verhandelt. EOSC ist in seiner Ausrichtung breiter als die NFDI, da es „Open Science“ als Gesamtkonzept verfolgt, während die NFDI auf Forschungsdaten fokussiert (s. o.). Beide setzen im Überbau auf fachspezifische Infrastrukturen, die als Konsortien organisiert sind. Die europäischen Research Infrastructures sind zum Teil in einer Rechtsform etabliert worden, vor allem als European Research Infrastructure Consortium (ERIC), während in Deutschland über die Hochschulen und außeruniversitären Forschungseinrichtungen institutionelle Rechtsformen existieren. Speziell für digitale Forschungsinfrastrukturen fördert Europa „e-Infrastructures“ als Projekte (teilweise mit Betriebsmitteln) in den Rahmenprogrammen, während in Deutschland - wie bei den Forschungsinfrastrukturen - die Finanzierung institutionell, beispielsweise in Rechenzentren und Bibliotheken, verankert ist.

Das Verhältnis zwischen einem Mitgliedsstaat, der seine Autonomie bewahrt, und der auf Zusammenarbeit ausgerichteten europäischen Idee lässt sich also deutlich an den Unterschieden in den politischen Prozessen und Steuerungs- sowie Finanzierungmodellen ablesen. Mit dem Wissenschaftsrat, dem RfII und der Allianz der Wissenschaftsorganisationen existieren eigene Beratungsgremien und Entscheidungen werden maßgeblich von der GWK und den Bundesländern getragen.

Zur Verknüpfung der mitgliedsstaatlichen Interessen mit den europäischen Initiativen existieren systematische Vernetzungen der europäischen und nationalen Aktivitäten: Forschungsgruppen, Bibliotheken und Rechenzentren der Mitgliedsstaaten können Partner in den europäischen Initiativen und ESFRIInfrastrukturen sein. Außerdem weisen die Mitgliedsstaaten häufig Finanzbei- 
träge für die ESFRI-Infrastrukturen $\mathrm{zu}$ und definieren Eigenanteile (in-kind contributions), die einem gemeinsamen europäischen Betriebskonzept dienen. Regierungen sind über Ministerien außerdem regelmäßig in den Steuerungsgremien einzelner ESFRI-Infrastrukturen beteiligt und entscheiden auch in der Gesamtheit über die Zusammenarbeit, z. B. im Competitiveness Council. Regierungen partizipieren ebenfalls an den Beratungsgremien für ESFRI, der e-Infrastructure Reflection Group (eIRG) und dem European Research Area and Innovation Committee (ERAC).

Tab. 1: Gegenüberstellung zentraler Begriffe für digitale Forschungsinfrastruktur in Deutschland und Europa (Beschreibung im Text, Referenzen am Ende des Beitrags).

\begin{tabular}{|c|c|c|}
\hline & Deutschland & Europa \\
\hline $\begin{array}{l}\text { Konzepte für Forschungs- } \\
\text { infrastrukturen }\end{array}$ & $\begin{array}{l}\text { BMBF-Roadmap für For- } \\
\text { schungsinfrastrukturen [1] }\end{array}$ & ESFRI-Roadmap [2] \\
\hline $\begin{array}{l}\text { Konzepte für digitale For- } \\
\text { schungsinfrastrukturen }\end{array}$ & NFDI [3] & EOSC [4] \\
\hline Implementierungsprojekte &.$/$ & $\begin{array}{l}\text { EOSCpilot [5], EOSC-hub [6], } \\
\text { OpenAIRE-Advance [7] }\end{array}$ \\
\hline $\begin{array}{l}\text { Fachspezifische digitale } \\
\text { Infrastrukturen }\end{array}$ & $\begin{array}{l}\text { in Hochschulen und } \\
\text { außeruniversitären } \\
\text { Forschungseinrichtungen } \\
\text { (vorgesehen: Konsortien der } \\
\text { NFDI) }\end{array}$ & $\begin{array}{l}\text { in „Research Infrastructures“ } \\
\text { (teilweise als ERIC-Rechtsform) }\end{array}$ \\
\hline $\begin{array}{l}\text { Fachübergreifende digitale } \\
\text { Infrastrukturen }\end{array}$ & $\begin{array}{l}\text { in Hochschulen und } \\
\text { außeruniversitären } \\
\text { Forschungseinrichtungen } \\
\text { (darin z. B. Bibliotheken, } \\
\text { Rechenzentren) }\end{array}$ & $\begin{array}{l}\text { „e-Infrastructures“ (nur IT und } \\
\text { Daten; digitale Wissensres- } \\
\text { sourcen nicht repräsentiert) }\end{array}$ \\
\hline Verbände & $\begin{array}{l}\text { Fachverbände, HRK [8], dbv } \\
\text { [9], ZKI [10], DINI [11] }\end{array}$ & $\begin{array}{l}\text { Learned Societies, EUA [12], LI- } \\
\text { BER [13], GÉANT [14] }\end{array}$ \\
\hline Beratungsgremien & $\begin{array}{l}\text { Wissenschaftsrat [15], } \\
\text { RfII [16], Allianz der Wissen- } \\
\text { schaftsorganisationen [17] }\end{array}$ & ERAC [18], ESFRI [19], elRG [20] \\
\hline Entscheidungsgremien & $\begin{array}{l}\text { KMK (GWK) [21], Bundes- } \\
\text { regierung }\end{array}$ & $\begin{array}{l}\text { EU-Rat (Competitiveness Coun- } \\
\text { cil) [22] }\end{array}$ \\
\hline Politisches Programm & $\begin{array}{l}\text { Digitale Agenda der Bundes- } \\
\text { regierung (wie Anm. 1) }\end{array}$ & $\begin{array}{l}\text { „Digital Single Market“ der Eu- } \\
\text { ropäischen Kommission (wie } \\
\text { Anm. 8) }\end{array}$ \\
\hline
\end{tabular}

Darüber hinaus existieren viele weitere Organisationen, deren Bedeutung nicht minder wichtig ist, deren Darstellung jedoch den vorliegenden Rahmen sprengen würde. Dazu gehören die vielfältigen Fachverbände, die Forschungsförderer, wie die DFG in Deutschland und Science Europe in Europa, die Hochschu- 
len mit der Hochschulrektorenkonferenz (HRK) und der European University Association (EUA), die Infrastruktureinrichtungen mit dem Deutschen Bibliotheksverband (dbv), den Zentren für Kommunikation und Informationsverarbeitung (ZKI) oder der Deutschen Initiative für Netzwerkinformation (DINI), dem europäischen Bibliotheksverband (LIBER) und dem europäischen Forschungsnetz GÉANT.

\section{Chancen und Risiken für wissenschaftliche Bibliotheken in digitalen Forschungsinfrastruk- turen}

Vor der Digitalisierung hatten wissenschaftliche Bibliotheken die zentrale Rolle als Informationsinfrastruktur für die Forschung. Ohne damit sagen zu wollen, dass es die einzige Möglichkeit für Forschende war, an Informationen zu kommen - Forschende haben immer auch andere Dienste genutzt -, gab es keine andere große, institutionalisierte Einrichtung, die dauerhaft die Gemeinsamkeiten der Informationsanforderungen fachübergreifend bündelte.

Rechenzentren sind in den letzten Dekaden ein wesentlicher und notwendiger Bestandteil der Informationsinfrastruktur für die Forschung geworden. Es ist jedoch nicht so, dass Rechenzentren ein Monopol auf digitale Infrastrukturen haben. Bibliotheken stellen mit der digitalen Literaturversorgung einen wesentlichen Bestandteil der digitalen Infrastruktur und spielen in vielen Fällen eine tragende Rolle bei weiteren digitalen Diensten wie digitaler Lehre, Kursen für digitale Fertigkeiten, administrativer Forschungsinformation und direkter Unterstützung der digitalen Forschung in Sachen Software oder Daten. Die Grenzen zwischen Bibliotheken und Rechenzentren sind im großen Maßstab der Digitalisierung fließend. Es gibt Bibliotheken, die unabhängig von Rechenzentren Netzwerk, Speicher- und Rechenkapazität aufbringen, und es gibt Rechenzentren, die die digitale Literaturversorgung übernehmen. Und es gibt verschiedene Mischformen, die von der Integration in eine gemeinsame Institution über gemeinsame Steuerungs- und Finanzierungsströme für kooperative Modelle in Teilbereichen bis hin zu arbeitsteiligen Ko-Existenzen reichen. Die genaue Form der Zusammenarbeit hängt kritisch von den lokalen Umgebungen ab und muss im Einzelfall entwickelt werden. Welche genaue Arbeitsteilung auch immer vorliegt - die Zusammenarbeit von Bibliotheken und Rechenzentren ist ein Muss, wenn digitale Forschungsinfrastruktur aufgebaut 
wird. Ein Wettbewerb zwischen Bibliotheken und Rechenzentren ist ein Nebenschauplatz, der Kraft kostet, die an anderer Stelle dringend benötigt wird.

Für die Digitalisierung von Forschungsinfrastruktur ist es essenziell, dass die Anforderungen der Forschung angemessen umgesetzt werden. Die Masse an vorliegender und entstehender Information sowie die damit verbundene Spezialisierung der Fächer sind weitaus größere Herausforderungen als die Frage der Verortung von den gemeinsamen, fachübergreifenden Anteilen der Infrastruktur in Bibliotheken und Rechenzentren. Die entscheidende Frage ist: Welches sind die gemeinsamen, fachübergreifenden Anteile der digitalen Forschungsinfrastruktur? Gibt es über Netzwerke, Speicher- bzw. Rechenkapazitäten und digitale Literatur hinaus Gemeinsamkeiten in den Anforderungen, die fachübergreifend erbracht werden können? Oder rechtfertigen die fachspezifischen Anforderungen sogar, dass selbst diese Dienste nicht mehr gebündelt, sondern redundant in den einzelnen Disziplinen erbracht werden?

Die Fragen nach dem Verhältnis von fachspezifischer zu fachübergreifender digitaler Infrastruktur sind unbeantwortet: Eine Vervielfachung von autonomen fachspezifischen Infrastrukturen erscheint als Lösung fragwürdig, da es angesichts der kontinuierlichen Spezialisierung der Fachgebiete perspektivisch zu einer unendlichen Anzahl von einzelnen Infrastrukturen führen würde. Der finanzielle Aufwand für die wiederholte Bereitstellung derselben Funktionen für jede dieser Infrastrukturen müsste gegenüber der Gesellschaft zu rechtfertigen sein. Die Gesellschaft erwartet jedoch von der Forschung die Lösung großer Menschheitsfragen, etwa des Klimawandels, unheilbarer Krankheiten, der Sicherheit des Arbeitsplatzes, der Verhinderung von Krieg und Gewalt oder der Erklärung des Bewusstseins. Eine Verinselung der Fächer, in der jedes Fach für sich spezialisierte Antworten formuliert, ist vor diesem Hintergrund schwer zu vermitteln. Auf der anderen Seite kann es keine vorgeschriebene Rezeptur für Forschung geben, die eine für die Innovationskraft notwendige Selbstbestimmung der Forschung einschränkt. Die Freiheit der Wissenschaft ist ein Gut, das nicht durch Reglementierung der Infrastrukturen gefährdet werden kann.

Für Bibliotheken ist die Herausforderung, zwischen fachübergreifenden und fachlichen Infrastrukturen $\mathrm{zu}$ vermitteln, größer als die der Herausforderung der Digitalisierung selbst. Die Digitalisierung ist nämlich in diesem Sinne ein Modus Operandi einer zunehmend vielfältiger werdenden Wissenschaft, die ständig neue fachliche Strukturen und neue Methoden entwickeln muss, um mit der Masse an Informationen umzugehen und diese zu interpretieren. Die Digitalisierung wird zum Sinnbild des Wandels, ist jedoch nicht der Wandel selbst.

Es kann erhellend wirken, sich auf den ursprünglichen Auftrag der wissenschaftlichen Bibliotheken zu besinnen: Wissensressourcen für Forschung (und 
Lehre) $\mathrm{zu}$ erschließen und zu managen. Wissensrepräsentationen sind heute nicht mehr nur Texte in Form eines analogen oder digitalen Buches, sondern auch Daten und Software. Diese jedoch erfordern völlig neue Kompetenzen in den Bibliotheken und ein tief greifendes Verständnis der Forschungsprozesse. Forschende wissen intuitiv, wie sie Daten und Software als Wissensressourcen benutzen, es ist jedoch nicht ihre Aufgabe, Bibliotheken zu erklären, wie dies dauerhaft infrastrukturell $\mathrm{zu}$ unterstützen ist. Bibliotheken haben eine Holschuld, sehr eng mit der Forschung zusammenzuarbeiten oder die Forschenden in die Bibliothek $\mathrm{zu}$ integrieren. Hierfür gibt es einen Präzedenzfall: Forschungsbibliothekare waren in der Zeit, als Wissensressourcen durch die Industrialisierung der Buchproduktion exponentiell anwuchsen, auch ein Mittel der Wahl, mit der plötzlich steigenden Komplexität umzugehen. Diese Rolle wurde durch die Einführung von Fachreferenten professionalisiert und dauerhaft in der Organisationsstruktur der Bibliothek verankert. Fachreferenten jedoch sind durch nach wie vor bestehende Erwerbungsaufgaben für Wissensressourcen in digitaler und analoger Textform gebunden. Eine Herausforderung ist also, die Ressourcen aufzubringen, Forschende in die Bibliothek zu integrieren, um neue Aufgaben wahrzunehmen.

Bei der Gewinnung von Forschenden für die Bibliotheken ist zu beachten, dass es nicht allein die fachliche Orientierung ist, die repräsentiert werden muss. Der Umgang mit Software und Daten erfordert methodische Kompetenzen, etwa der statistischen Analyse, der Programmierung oder der Pflege von Ontologien, Vokabularien oder quantitativen Datenstrukturen zur Wissensorganisation. Digitalisierung verändert also nicht den Auftrag der Bibliothek, Wissensressourcen zu organisieren, verstärkt jedoch den Bedarf für Dienste in einer neuen Dimension der Funktion von Bibliotheken: die der Methoden. Die Hilfswissenschaften der Geisteswissenschaften können hier als Vergleich dienen.

Ein Weg, die Herausforderung der Integration von Forschenden in die Bibliothek anzugehen, sind gemeinsame Drittmittelprojekte. Dies ist ein probates Mittel, das mehr und mehr in Bibliotheken praktiziert wird, stellt jedoch lediglich eine temporäre Lösung dar. Forschung und Bibliothek sind hier in derselben Situation. Auch die Forschung steht vor der Aufgabe, die neuen Wissensressourcen in ein dauerhaftes, infrastrukturelles Angebot zu überführen, und fordert von Forschungsförderern zusätzliche und dedizierte Mittel, dies umzusetzen. Und die Wissenschaftspolitik ihrerseits fordert die Offenheit von Wissensressourcen. Wenn man „Open Science“ als Auftrag versteht, eine Infrastruktur zu finden, die die immer vielfältiger und größer werdenden Wissensressourcen aller wissenschaftlichen Disziplinen und der gesamten Gesellschaft 
dauerhaft, einfach und verständlich zugreifbar macht, ergibt sich also ein Konsens zwischen Bibliothek, Forschung und Politik.

Zusammengenommen ist ebenso nachvollziehbar, dass der RfII auf das Thema Forschungsdaten kapriziert und dass die europäische Politik auf das Thema Offenheit abzielt, denn beide sind Aspekte, die tatsächlich eine Gemeinsamkeit der Bedarfe von Wissenschaft, Gesellschaft und Bibliotheken abbilden. Die Rolle von Bibliotheken in digitalen Infrastrukturen ist also nicht neu: Bibliotheken sind ein Filter in einer immer komplexeren Informationswelt, der Methoden der Wissensorganisation dauerhaft organisational verankert. Es liegt nun an den Bibliotheken, diese eigentlich traditionelle Rolle neu zu interpretieren.

\section{Webseiten zu Tab. 1 (alle zuletzt am 5.2.2018 aufgerufen)}

BMBF-Roadmap für Forschungsinfrastrukturen [1]: https://www.bmbf.de/de/roadmap-fuerforschungsinfrastrukturen-541.html

ESFRI-Roadmap [2]: http://www.esfri.eu/roadmap-2018

NFDI [3]: https://www.bmbf.de/de/empfehlungen-zum-management-von-forschungsdaten3036.html

EOSC [4]: https://ec.europa.eu/research/openscience/index.cfm?pg=open-science-cloud EOSCpilot [5]: https://eoscpilot.eu/content/stakeholders-view-eosc-service-portfolio-

management

EOSC-hub [6]: https://www.egi.eu/about/newsletters/introducing-the-eosc-hub-project/

OpenAIRE-Advance [7]: https://www.openaire.eu/advance

HRK [8]: https://www.hrk.de

dbv [9]: http://www.bibliotheksverband.de

ZKI [10]: https://www.zki.de

DINI [11]: https://www.dini.de/startseite/

EUA [12]: http://www.eua.be

LIBER [13]: http://libereurope.eu

GÉANT [14]: https://www.geant.org

Wissenschaftsrat [15]: https://wissenschaftsrat.de/home.html

RfIl [16]: http://www.rfii.de/de/index/

Allianz der Wissenschaftsorganisationen [17]: https://www.allianzinitiative.de

ERAC [18]: http://www.consilium.europa.eu/de/council-eu/preparatory-bodies/european-

research-area-innovation-committee/

ESFRI [19]: http://www.esfri.eu

eIRG [20]: http://e-irg.eu

KMK (GWK) [21]: http://www.gwk-bonn.de

EU-Rat (Competitiveness Council) [22]: http://www.consilium.europa.eu/en/council-eu/configurations/compet/ 